\title{
Investigation of avian haemosporidian parasites from raptor birds in Turkey, with molecular characterisation and microscopic confirmation
}

\author{
Arif Ciloglu ${ }^{1}$, Alparslan Yildirim ${ }^{1}$, Onder Duzlu ${ }^{1}$, Zuhal Onder $^{1}$, Zafer Dogan $^{2}$ and Abdullah Inci ${ }^{1}$ \\ ${ }^{1}$ Department of Parasitology, Faculty of Veterinary Medicine, Erciyes University, Kayseri, Turkey; \\ ${ }^{2}$ Department of Surgery, Faculty of Veterinary Medicine, Erciyes University, Kayseri, Turkey
}

\begin{abstract}
Avian haemosporidians are common vector-borne blood parasites that have been reported in birds all over the world. Investigations of avian haemosporidian parasites are conducted mainly on passerine birds. However, studies that focus on non-passerine avian hosts are important for our understanding of the true diversity, host specificity and genetic variability among these widespread parasites. In the present study, blood samples from a total of 22 raptor birds belonging to two orders, two families and six species from the Central Anatolia Region of Turkey were investigated for three genera of avian haemosporidians (Plasmodium Marchiafava et Celli, 1885, Haemoproteus Kruse, 1890 and Leucocytozoon Sambon, 1908) using a combination of microscopic examination of blood films and nested PCR targeting the parasite mitochondrial cytochrome $b$ gene (cyt- $b$ ). In total, six individual raptor birds identified positive for species of Plasmodium or Leucocytozoon and one individual was found co-infected with all three haemosporidian genera. We identified five parasite cyt- $b$ haplotypes, three of which were reported for the first time. Among these, one Plasmodium haplotype is linked to a corresponding morphospecies (P-TURDUS1, Plasmodium circumflexum Kikuth, 1931). All haplotypes were clearly distinguishable in phylogenetic analyses. As one of the first studies to investigate blood parasites from non-passerine birds in the Central Anatolia Region of Turkey, this study provides important new information on the phylogenetic relationships and genetic diversity of avian haemosporidian parasites from raptor birds. We discuss these findings in the context of avian haemosporidian host-parasite relationships and we draw attention to the need for microscopy to detect parasite sexual development stages in surveys of avian haemosporidians.
\end{abstract}

Keywords: Plasmodium, Haemoproteus, Leucocytozoon, Accipitriformes, Strigiformes, cytochrome $b$ gene, blood parasites

Avian haemosporidians (Apicomplexa: Haemosporida) belonging to the families Haemoproteidae, Plasmodiidae and Leucocytozoidae are vector-borne parasites that are found in birds all over the world. Avian haemosporidian parasites are transmitted by biting midges, louse flies, black flies and mosquitoes (Valkiūnas 2005). More than 250 species of avian haemosporidians have been described and named according to morphological characteristics of parasite blood stages, and over 2000 unique genetic haplotypes have been identified and recorded by molecular studies (Valkiūnas 2005, Bensch et al. 2009, Clark et al. 2014). Because these blood parasites are abundant and diverse, they constitute excellent model organisms for the study of wildlife parasitology and have been the focus of research efforts for over 100 years (Valkiūnas 2005, Bensch et al. 2013).

Raptor birds (Accipitriformes and Strigiformes) are located at the top of the food chain and can play very important roles in the ecosystem. However, most studies of avian haemosporidian parasites have been conducted on passerines (Passeriformes), with very little focus on raptor hosts (Krone et al. 2008, Clark et al. 2014). More studies are crucial to improve our understanding of the true diversity, genetic variability and host specificity of blood parasites in raptor birds, particularly since raptor hosts are highly diverse and can support cryptic speciation of avian haemosporidians (Sehgal et al. 2006, Pérez-Rodríguez et al. 2013). Moreover, while the blood parasites are generally thought to be harmless, there is evidence that infections can be harmful (Remple 2004). Studies have suggested that avian haemosporidian infection can cause reduced speed, reduced strength in flight, poor appetite, weight loss, anemia, airsacculitis and arthritis in hosts (Remple 1981, 2004, Dawson and Bortolotti 2000, Merino et al. 2000). Thus, from a conservation perspective, the diagnosis and treatment of avian haemosporidian infections are important for controlling the cumulative effects of blood parasites in raptor birds.

Turkey is home to a high diversity of birds and is considered one of the most important regions in Europe and the Middle East for migratory birds that travel through Africa, Europe and Asia (Yigit et al. 2008). However, there are very few studies on avian haemosporidians and their vectors in this geographic region (Inci et al. 2012, 2013). Moreover, most avian haemosporidian investigations 
Table 1. Raptor bird species and infection results from microscopic examination of blood films and PCR screening. GenBank accession numbers of parasite haplotypes are given in parentheses.

\begin{tabular}{|c|c|c|c|c|c|c|c|c|c|c|c|c|}
\hline \multirow[b]{2}{*}{ Raptor species } & \multirow[b]{2}{*}{$\begin{array}{l}\text { No. } \\
\text { tested }\end{array}$} & \multicolumn{5}{|c|}{$\begin{array}{l}\text { Positive in microscopic } \\
\text { examination }\end{array}$} & \multicolumn{6}{|c|}{ Positive in PCR screening } \\
\hline & & $\mathrm{H}$ & $\mathrm{L}$ & $\mathrm{P}$ & $\begin{array}{c}\text { No. } \\
\text { co-infected } \\
(\mathrm{H}, \mathrm{L}, \mathrm{P})\end{array}$ & Total & $\mathrm{H}$ & $\mathrm{L}$ & $\mathrm{P}$ & $\begin{array}{l}\text { No. } \\
\text { co-infected } \\
(\mathrm{L}, \mathrm{P})\end{array}$ & Total & $\begin{array}{l}\text { Parasite haplotypes with } \\
\text { GenBank Accession Nos. }\end{array}$ \\
\hline Accipiter nisus (Linnaeus) & 1 & - & - & 1 & - & 1 & - & - & 1 & - & 1 & P-BUTBUT02¹ (KP883280) \\
\hline Asio otus (Linnaeus) & 1 & - & - & - & - & - & - & 1 & - & - & 1 & L-CIAE02 (KP000840) \\
\hline Buteo buteo (Linnaeus) & 5 & - & 1 & 1 & 1 & 3 & - & 1 & 1 & 1 & 3 & $\begin{array}{l}\text { P-BUTBUT02 }{ }^{1} \text { (KP883279), } \\
\text { P-TURDUS1 (KP000842), } \\
\text { L-BUTBUT01 }{ }^{1} \text { (KP000841), } \\
\text { L-CIAE02 (KC962152) }\end{array}$ \\
\hline Buteo rufinus Cretzschmar & 13 & - & 1 & - & - & 1 & - & 1 & - & - & 1 & L-CIAE02 (KC962151) \\
\hline Neophron percnopterus (Linnaeus) & 1 & - & - & - & - & - & - & - & - & - & - & - \\
\hline Strix aluco Linnaeus & 1 & - & 1 & - & - & 1 & - & 1 & - & - & 1 & L-STAL5 ${ }^{1}$ (KC876042) \\
\hline Total & 22 & - & 3 & 2 & 1 & 6 & - & 4 & 2 & 1 & 7 & - \\
\hline
\end{tabular}

$\mathrm{H}$ - Haemoproteus sp.; L - Leucocytozoon sp.; P-Plasmodium sp.; ${ }^{1}$ newly described haplotypes.

that have been carried out in Turkey have relied entirely on traditional microscopic examinations (Özmen et al. 2005, 2009). According to our knowledge, there are only two published molecular surveys of avian haemosporidians in Turkey, one that focused on the Krüper's nuthatch (Sitta krueperi Pelzeln) and another that sampled a tawny owl (Strix aluco Linnaeus) (Marzal and Albayrak 2012, Yildirim et al. 2013).

The ability to detect avian haemosporidians in vertebrate hosts has been improved recently with the advent of molecular-based screening techniques (Bensch et al. 2000, Ricklefs and Fallon 2002, Beadell et al. 2004). In addition, our understanding of the genetic diversity and host specificity of avian haemosporidians has been vastly improved through molecular surveys (Hellgren et al. 2009, Ventim et al. 2012) and these methods have facilitated the description of numerous parasite DNA sequences that have been deposited in GenBank and the MalAvi databases (Ricklefs et al. 2004, Waldenström et al. 2004, Bensch et al. 2009, MalAvi 2015). Nevertheless, although PCR-based methods have very low detection thresholds and are considered more sensitive than traditional parasitological methods, microscopic analyses remain essential for understanding the true diversity and host specificity of avian haemosporidians (Valkiūnas et al. 2009, Clark et al. 2015). It is therefore important to combine microscopic and molecular surveys for characterising avian haemosporidians in wild avian hosts (Valkiūnas et al. 2009, Clark et al. 2014).

In the present study, we aim to investigate avian haemosporidian parasites in accipitriform and strigiform host samples from the Central Anatolia Region of Turkey. We combine both traditional microscopy and molecular-based techniques in order to carry out one of the first descriptions of the phylogenetic relationships, haplotype diversity and host specificity of haemosporidian parasites from raptor birds.

\section{MATERIALS AND METHODS}

We sampled injured and/or sick individual accipitriform and strigiform birds that were found in Kayseri Province or near cities in the Central Anatolia Region of Turkey. All sampled birds were brought to the Surgery Department of Veterinary Education Research and Practice Hospital of Erciyes University, Kayseri for treatment and rehabilitation between February 2013 and June 2014. Blood samples were taken from vena cutanea ulnaris of 20 accipitriform and 2 strigiform hosts using insulin syringes for investigating the presence of haemosporidian parasites during the hospitalisation process (Table 1). About 50-100 $\mu$ l of blood was taken and stored in EDTA (ethylenediaminetetraacetic acid) tubes and immediately placed at $-20^{\circ} \mathrm{C}$ for DNA extractions. Immediately after puncturing the vein, at least two thin blood films were prepared on clean glass slides from each bird and air-dried within 5-10 s after their preparation. Blood films were fixed in $100 \%$ methanol and stained with $10 \%$ Giemsa as described by Valkiūnas et al. (2008a).

Microscopic examinations were carried out to distinguish between parasite sexual or asexual blood stages, a determination that cannot be made by PCR amplification. Each blood film was carefully examined using an Olympus BX43 light microscope (Olympus, Tokyo, Japan) equipped with Olympus DP73 digital camera. At least 100 fields were examined under $400 \times$ and $1000 \times$ magnification for 30-45 min. Morphological identification of parasites was performed according to Valkiūnas (2005).

Prior to DNA extraction, $10-15 \mu 1$ of blood from each sample was diluted with $200 \mu \mathrm{l}$ of PBS buffer $(0.01 \mathrm{M}$ phosphate buffer, $0.0027 \mathrm{M} \mathrm{KCl}, 0.137 \mathrm{M} \mathrm{NaCl}, \mathrm{pH}$ 7.4). Total genomic DNA (gDNA) was extracted from blood samples using Blood Genomic DNA Kits (AP-MN-BL-GDNA-250, Axygen Biosciences, Tewksbury, MA, USA) and eluted in $50 \mu$ l elution buffer following the manufacturer's instructions. The isolated gDNA was stored at $-20^{\circ} \mathrm{C}$ until molecular analysis. DNA concentrations of samples were measured using the Nano Drop Spectrophotometer (ASP-3700, ACT Gene, Piscataway, NJ, USA) prior to molecular analyses to standardise the amount of gDNA used in PCR amplifications.

Genomic DNA from blood samples was analysed by nested PCR method for amplification of the cytochrome $b$ gene (cyt- $b$ ) of avian haemosporidian mitochondrial DNA (mtDNA). For the first amplification, we used primers HaemNFI and HaemNR3, which were designed to detect DNA from species of Leucocytozoon Sambon, 1908, Haemoproteus Kruse, 1890 and Plasmodium Marchiafava et Celli, 1885 (see Hellgren et al. 2004). For 
the second PCR round, HAEMF-HAEMR2 primers (Bensch et al. 2000) and HaemFL-HaemR2L primers (Hellgren et al. 2004) were used for the amplification of species of Haemoproteus/Plasmodium and Leucocytozoon, respectively. The first PCR reaction was performed in a total volume of $25 \mu \mathrm{l}$, composed of $12.5 \mu \mathrm{l}$ of commercial Master Mix (Maxima Hot Start PCR Master Mix, Thermo Scientific, Waltham, MA, USA), $10 \mu \mathrm{M}$ concentrations each primer and $50 \mathrm{ng}$ of genomic DNA in C1000 Thermal Cycler (Bio-Rad, Hercules, CA, USA). For the second PCR, $1 \mu \mathrm{l}$ of the first PCR's product was used as template. PCR cycling profiles followed Hellgren et al. (2004). Amplification products $(10 \mu \mathrm{l})$ were analysed by gel electrophoresis in $1.5 \%$ agarose containing ethidium bromide $(0.5 \mu \mathrm{g} / \mathrm{ml})$. The amplicons were visualised with UV illumination and imaged on a CLP Gel Documentation System (UVP INC Uplant, CA, USA). Two positive controls (Haemoproteus sp. and Leucocytozoon sp. which were provided by Department of Parasitology, Faculty of Veterinary Medicine, Erciyes University, Kayseri, Turkey and Department of Biology, Molecular Ecology and Evolution Laboratory, Lund University, Lund, Sweden, respectively) and one negative control (sterilised-deionised water) were used in all PCR amplifications. All samples were analysed twice for avian haemosporidians by nested PCR.

Samples exhibiting positive amplification were purified for sequencing using the High Pure PCR product purification kit (Roche, Mannheim, Germany). The cyt- $b$ target fragment was sequenced in both directions using HAEMF-HAEMR2 primers for species of Plasmodium and Haemoproteus spp. and HaemFL-HaemR2L primers for Leucocytozoon (Macrogen, Amsterdam, The Netherlands). Sequences were edited in Geneious R8 (Kearse et al. 2012). The sequences were aligned to homologues available from GenBank using the BLASTn algorithm with the default settings. We calculated sequence divergences between different haplotypes using a Kimura 2-parameter substitution model (Kimura 1980) implemented in the program MEGA 6 (Tamura et al. 2013). The final cyt- $b$ haplotypes were identified and named (as a haplotype name) following the nomenclature in the MalAvi database. Unique nucleotide sequences generated in this study were deposited in GenBank with accession nos. KC876042, KC962151-2, KP000840-2 and KP883279-80.

Sequences of this study along with several haplotype sequences from species of Plasmodium and Leucocytozoon available in the MalAvi or GenBank databases were used in the construction of phylogenetic trees. The phylogenetic tree was constructed using Maximum Likelihood (ML) analysis based on the Kimura 2-parameter model (Kimura 1980) in the program MEGA 6 (Tamura et al. 2013). We performed 1000 bootstrap iterations for each tree. We obtained initial trees for the heuristic search by applying BioNJ and Neighbor-Join algorithms to a matrix of pairwise distances estimated using the Maximum Composite Likelihood (MCL) approach and selecting the appropriate topology based on log likelihood values. A phylogenetic tree (Fig. 3) reconstruction was also performed by Bayesian inference. The Bayesian analysis was run in MrBayes version 3.2.6 (Huelsenbeck and Ronquist 2001) through the Geneious R8 (Kearse et al. 2012) software in order to compare the obtained sequences with the haplotype sequences from species of Plasmodium that were microscopically identified and information is available in the MalAvi and GenBank databases. The General Time Revers- ible Model including invariable sites and variation among sites $(\mathrm{GTR}+\mathrm{I}+\mathrm{G})$ was utilised according to the results of the Akaike's Information Criterion in jModelTest version 0.1.1 (Posada 2008). Two Markov Chain Monte Carlo (MCMC) simulations were run simultaneously for 10 million generations with sampling every 200 generations. Before constructing a majority consensus tree, $25 \%$ of the initial trees in each run were discarded as burn-in periods. The available haplotype sequences from morphologically-described species of Leucocytozoon from raptor birds in the MalAvi and GenBank databases were amplified by using different primer pairs targeted different fragment of the parasite cyt- $b$ gene. Thus, these sequences could not be used in the phylogenetic constructions along with the obtained sequences from Leucocytozoon haplotypes in this study.

\section{RESULTS}

\section{Morphological analysis}

Two birds from the order Accipitriformes were infected with Plasmodium spp. and three birds (two from Accipitriformes and one from Strigiformes) were infected with Leucocytozoon spp. By microscopic examination, one bird from Accipitriformes was found to be co-infected with haemosporidians belonging to all three parasite genera (Haemoproteus, Leucocytozoon and Plasmodium) (Table 1). In birds that were positive for infection with Leucocytozoon spp., fusiform-shaped gametocytes were found in all blood films (Fig. 1A,B). The Haemoproteus sp. infection was characterised by gametocytes that were closely appressed to the nucleus of infected erythrocytes (Fig. 1C). For infection with Plasmodium spp., roundish and/or irregular shaped trophozoites were seen in infected mature erythrocytes in positive blood films (Fig. 1D).

\section{Molecular analysis}

Seven of the 22 blood samples yielded positive PCR amplifications, whereas six blood samples were found positive by microscopic examinations (Table 1). By combining results from both methods, the overall prevalence of infections for the 22 sampled raptor birds was 32\%. One Leucocytozoon spp. PCR-positive sample could not be observed in its corresponding blood films by microscopic examination. In addition, one sample that produced positive amplifications for both Plasmodium sp. and Leucocytozoon sp. by PCR was also found to be infected with a Haemoproteus sp. using microscopy, an infection that could not be detected by PCR. The cyt- $b$ of the parasite mtDNA target fragment was successfully amplified and sequenced from all PCR positive samples, and all sequences were submitted to the MalAvi and GenBank databases. In total, five different parasite haplotypes from two avian haemosporidian genera were found, three of which had not been previously described (Table 1). The Leucocytozoon haplotypes L-CIAE02 and L-BUTBUT01 were identified in individuals from Buteo rufinus Cretzschmar and Buteo buteo (Linnaeus) (order Accipitriformes), with L-BUTBUT01 constituting a new haplotype in the MalAvi and GenBank databases. We also found haplotype L-CIAE02 along with one other Leucocytozoon haplotype (L-STAL5) infecting strigiform 

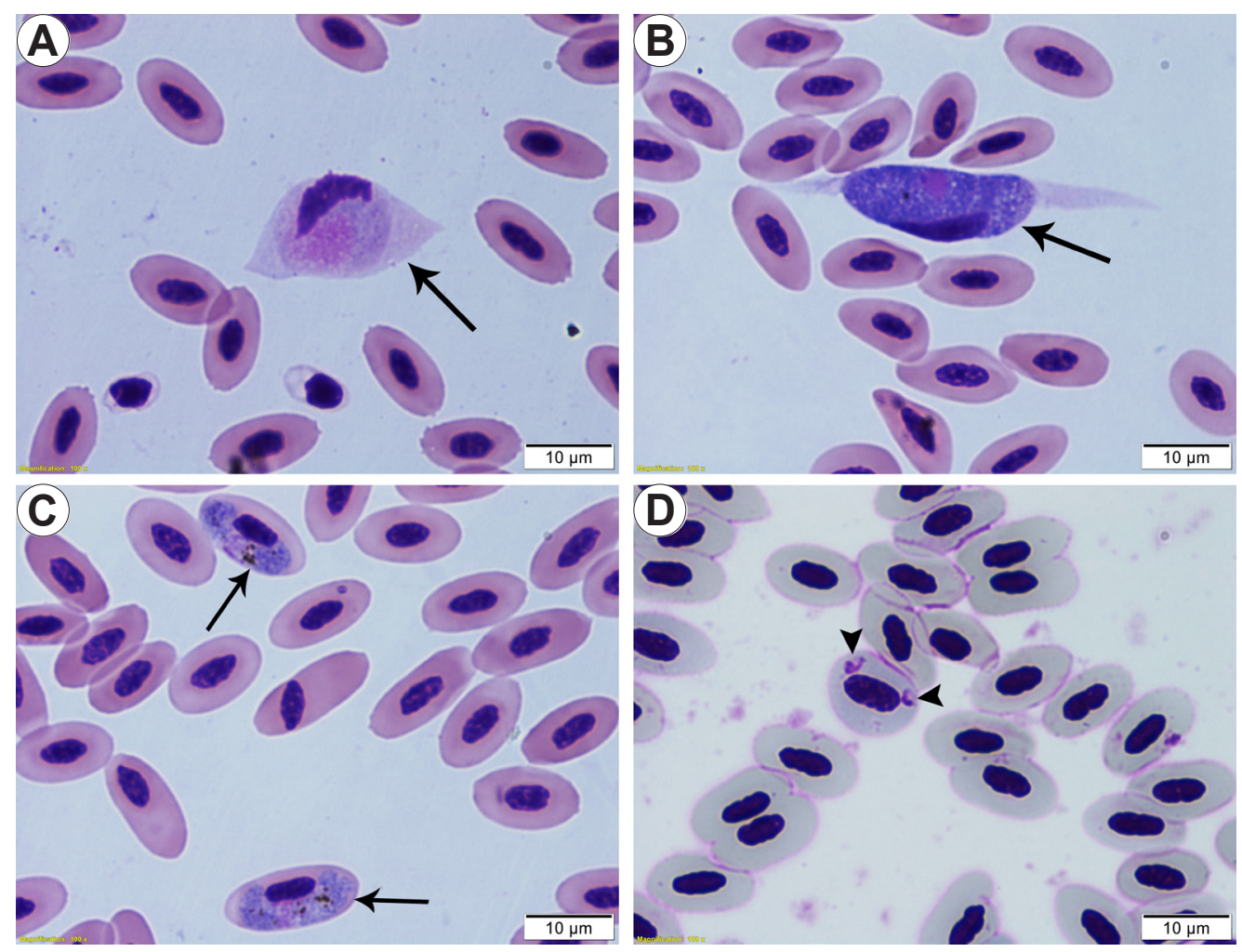

Fig. 1. Giemsa-stained thin blood films of erythrocytic stages of avian haemosporidian parasites (species of Leucocytozoon, Haemoproteus and Plasmodium) from the blood of examined raptor birds. A - microgametocyte of Leucocytozoon sp. from Buteo buteo; B - macrogametocyte of Leucocytozoon sp. from Buteo rufinus; C - macrogametocytes of Haemoproteus sp. from B. buteo; D - trophozoites of Plasmodium sp. from Accipiter nisus. Arrows indicate the sexual stages of parasites and arrowheads show trophozoites of Plasmodium sp.

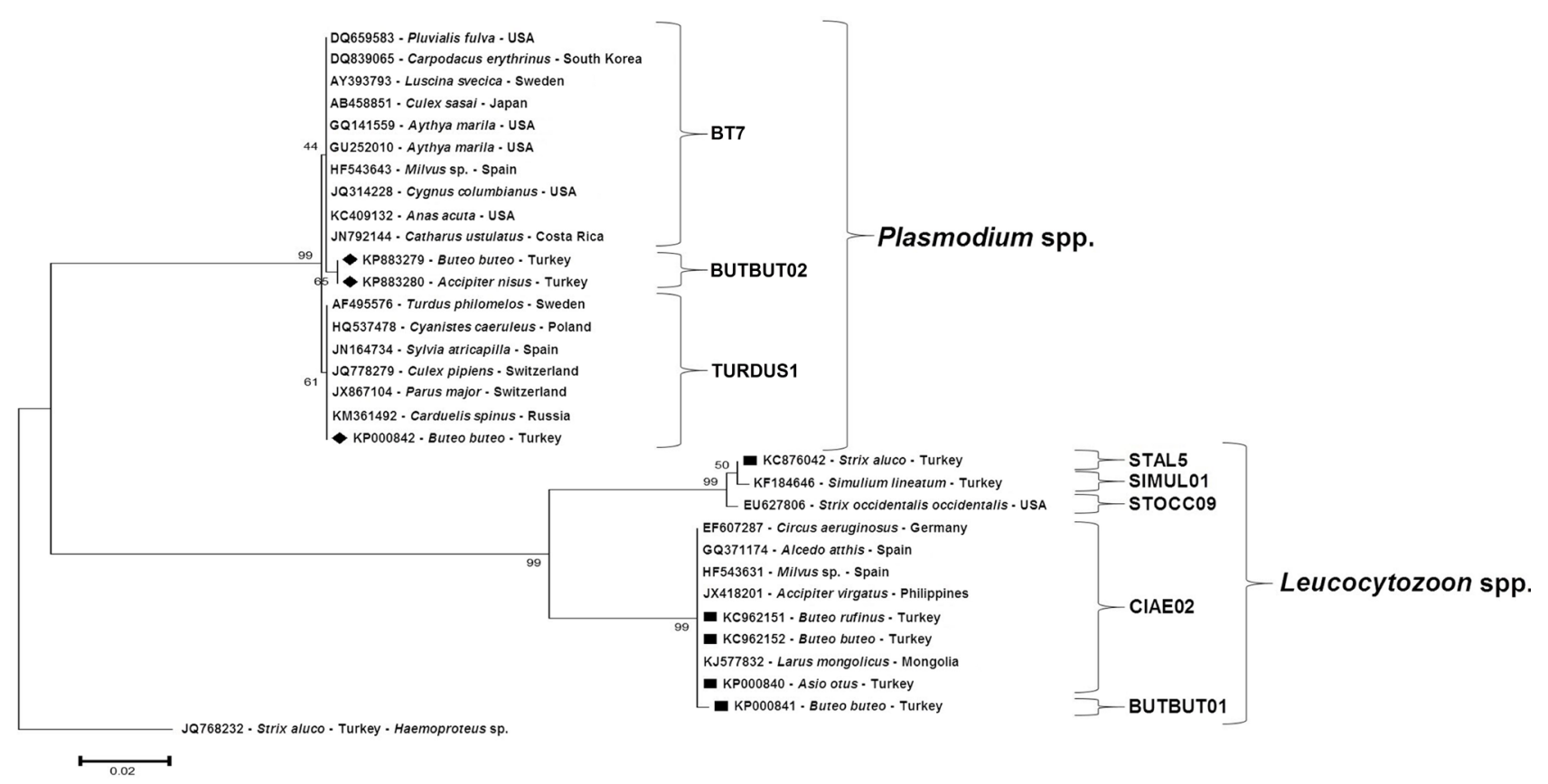

Fig. 2. Phylogenetic relationships among the obtained haemosporidian parasite haplotypes (denoted by symbols) and previously published haplotypes as inferred from partial cytochrome $b$ (cyt- $b$ ) sequences. Sequence names give GenBank accession number, host species and country in which the haplotype was recorded. Haplotype nomenclature follows the protocols of the MalAvi database (http://mbio-serv2.mbioekol.lu.se/Malavi). Numbers above branches indicate maximum likelihood bootstrap support (1000 replicates). The tree is drawn to scale, with branch lengths measured in the number of substitutions per site. One Haemoproteus sp. (GenBank accession no. JQ768232) cyt- $b$ haplotype was used as an outgroup. 


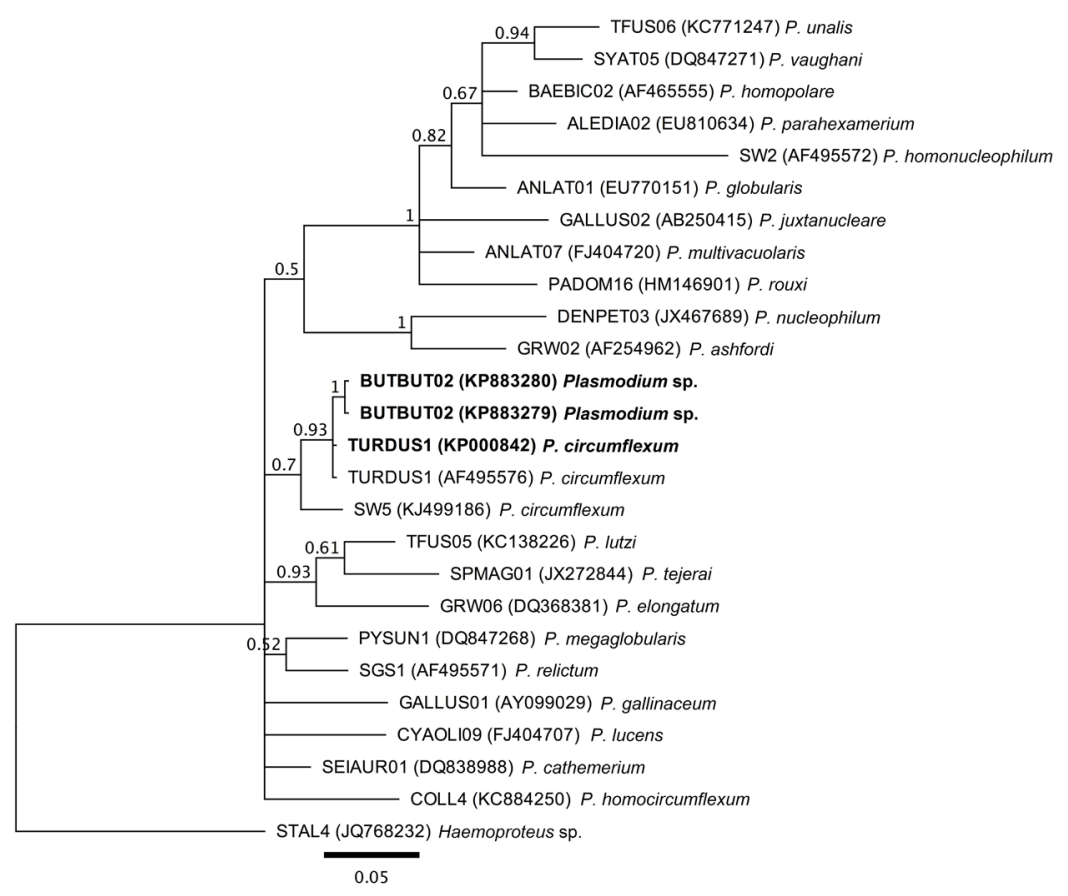

Fig. 3. Bayesian phylogeny of 23 cytochrome $b$ (cyt-b) haplotypes of avian Plasmodium spp. Haplotypes recorded in this study are given in bold. Haplotype nomenclature follows the protocols of the MalAvi database (http://mbio-serv2.mbioekol.lu.se/Malavi). GenBank accession numbers are shown in parentheses. Nodal support values near branches indicate posterior clade probabilities. The tree is drawn to scale, with branch lengths measured in the number of substitutions per site. One Haemoproteus sp. cyt-b haplotype was used as outgroup.

birds - Asio otus (Linnaeus) and Strix aluco Linnaeus. Although haplotype L-CIAE02 had already been found in birds belonging to the orders Charadriiformes, Gruiformes and Coraciiformes, L-STAL5 was described for the first time in a strigiform bird (S. aluco). For Plasmodium spp., haplotypes P-TURDUS1 and P-BUTBUT02 were found in accipitriform birds. Haplotype P-BUTBUT02, which was found infecting B. buteo and Accipiter nisus (Linnaeus), constituted a new haplotype in the MalAvi and GenBank databases.

\section{Phylogenetic analysis}

Individual sequences from P-BUTBUT02, L-STAL5 and L-BUTBUT01 haplotypes were clearly distinguishable in the phylogenetic tree (Fig. 2). P-TURDUS1 and L-CIAE02 haplotypes clustered together with haplotypes of parasites obtained from mosquito vectors in Switzerland and Japan as well as with several haplotypes reported from avian hosts belonging to several different orders. To further characterise our Plasmodium infections, we carried out a phylogenetic analysis that included sequences that were previously linked to Plasmodium morphospecies. The haplotypes of morphological species were all clearly distinct in this phylogenetic tree and our newly described haplotype P-BUTBUT02 clustered together with haplotypes previously linked to the morphospecies Plasmodium circumflexum Kikuth, 1931 (Fig. 3). Haplotype P-BUTBUT02 showed $99.5 \%$ and $97.4 \%$ identity to P-TURDUS1 and SW5 haplotypes, respectively, both of which were morphologically described as $P$. circumflexum by Palinauskas et al. (2007) and Valkiūnas et al. (2014).
P-BUTBUT02 and P-TURDUS1 haplotypes showed the most genetic similarity (99.8\%) to haplotype P-BT7. Haplotype L-STAL5 showed 99.8\%, 99.5\%, 92.8\% and 92.5\% identity to haplotype L-SIMUL01 obtained from Simulium lineatum Meigen in Turkey (GenBank accession no. KF184646), haplotype L-STOCC09 recorded in Strix occidentalis occidentalis Xantus de Vesey from USA (GenBank accession no. EU627806), and haplotypes L-CIAE02 and L-BUTBUT01, respectively. L-CIAE02 and L-BUTBUT01 were $99.8 \%$ identical to each other.

\section{DISCUSSION}

In the present study, both microscopy and PCR-based methods were applied to survey raptor birds for avian haemosporidian infection in Turkey. The main reason for using microscopy was to detect sexual development stages of avian haemosporidians in blood films. The sexual stages were observed in blood films from six out of seven PCR-positive samples. Although PCR amplified Leucocytozoon DNA from a blood sample from Asio otus, the sexual development stages of this infection could not be found in corresponding blood films. PCR-based method showed one more sample positive than microscopy. In contrast, one co-infected sample amplified DNA from Plasmodium and Leucocytozoon spp. but failed to amplify DNA from a Haemoproteus sp. that was clearly observed by microscopy. Moreover, gametocytes of Haemoproteus sp. from this particular bird were noticeably more abundant than erythrocytic stages of Plasmodium sp. This result supports the suggestion that co-infections with species of Haemoproteus and Plasmodium are not efficiently detected by cur- 
rent PCR techniques and that the use of double nucleotide peaks on sequence electropherograms to identify co-infections is insufficient (Valkiūnas et al. 2006, Martínez et al. 2009). This may explain why several former molecular studies have stated that single haemosporidian infections are more common than co-infections (Beadell et al. 2006, Bonneaud et al. 2009, Loiseau et al. 2010, Zehtindjiev et al. 2013). Hence, our results show that the combination of microscopy and PCR is essential for detection and identification of avian haemosporidians.

A portion of the mitochondrial cytochrome $b$ gene has often been used as a dominant gene region in taxonomy, systematics, ecology, biogeography and evolution studies of avian haemosporidian parasites (Bensch et al. 2009). For instance, Clark et al. (2014) found that 153 out of 162 examined avian haemosporidian publications targeted cyt- $b$ for nested PCR screening. Although different nuclear markers have been used in both host and vector studies, cyt- $b$ gene has been successfully used as a barcode of avian haemosporidian species to determine mtDNA lineages (Bensch et al. 2009, Chagas et al. 2013). Due to the lack of detailed information for many GenBank entries, the MalAvi database, which includes data on avian parasites belonging to the genera Plasmodium, Haemoproteus and Leuco$c y$ tozoon identified by a partial region of cyt- $b$ sequences, is a primary resource for describing patterns in the diversity and distribution of these parasites (Valkiūnas et al. 2008b, Bensch et al. 2009). In the present study, a 479 bp fragment of the cyt- $b$ gene was successfully used in order to define unique haemosporidian lineages. In phylogenetic analysis, we included both GenBank as well as MalAvi records for reducing the risk of using multiple names for identical lineages.

Species of avian Plasmodium are known to be host generalists and are often capable of being transmitted to avian hosts belonging to different families or orders (Waldenström et al. 2002, Križanauskienè et al. 2006, Dimitrov et al. 2010). However, species of Leucocytozoon are considered host-specific mostly at avian order and in some cases family, subfamily and species levels (Forrester and Greiner 2008). In the present study, P-BUTBUT02, L-STAL5 and L-BUTBUT01 were new haplotypes in the MalAvi and GenBank databases from all recorded haemosporidian haplotypes (5 in total). The remaining two haplotypes, P-TURDUS1 and L-CIAE02, have been known from previous studies. P-TURDUS1, which was described morphologically as P. circumflexum by Palinauskas et al. (2007), has been recorded in a variety of birds belonging to the orders Passeriformes, Accipitriformes and Charadriiformes (MalAvi 2015). Moreover, Krone et al. (2008) and Hanel et al. (2016) also reported P-TURDUS1 in Accipiter gentilis (Linnaeus) and Buteo buteo.

The results of our study support the statement that species of Plasmodium are host generalist and can infect hosts of wide range. Although L-CIAE02 has been found mainly in accipitriform birds, this haplotype was also recorded from several bird species belonging to the orders Charadriiformes, Gruiformes and Coraciiformes according to the MalAvi and GenBank databases. However, the studies which claimed that L-CIAE02 was found in different avian orders were generally unpublished, according to the available data on L-CIAE02 haplotypes. Moreover, Fourcade et al. (2014) found the L-CIAE02 haplotype in two infected corncrakes (Crex crex Linnaeus) using PCR-based methods in Poland and Russia, though no microscopy was included. However, in order to determine the true host specificity of this species of Leucocytozoon, it was necessary to make blood films and to do morphological analyses for detection of sexual development stages. Hence, due to the lack of information on the recorded sequences and also to differences in methodology across studies, it is difficult to claim that L-CIAE02 can complete its life cycle and produce infective stages in several different avian orders.

In the present study, haplotype L-CIAE02 was found in Buteo rufinus, B. buteo and A. otus. L-BUTBUT01 haplotype which differs from L-CIAE02 by a single nucleotide has been haplotyped for the first time from B. buteo. Another haplotype, L-STAL5 was found in Strix aluco and was closely related to another haplotype of Leucocytozoon (L-STOCC09) that was previously obtained from Strix occidentalis occidentalis. L-CIAE02 haplotype was recorded in $A$. otus by PCR, but sexual development stages could not be observed in blood films of this raptor bird even after careful examination of all blood films. It is possible that developmental stages could not be detected because of a very light infection. The other possibility is that PCR amplified DNA of persisted sporozoites in peripheral bloodstream that were recently injected by dipteran vector. Therefore, it is unclear if Leucocytozoon sp. (L-CIAE02) completes development in $A$. otus, a bird that belongs to the order Strigiformes. Although our results support the idea that species of Leucocytozoon can be host specific at the avian order level, it is still necessary to design experimental investigations using both avian and vectors hosts in order to understand the mechanism of host specificity of Leucocytozoon.

In the present study, the genetic distance between the newly recorded P-BUTBUT02 and P-TURDUS1 (P. circumflexum) was calculated as $0.5 \%$ and P-BUTBUT02 clustered together with haplotypes of $P$. circumflexum (Fig. 3). According to the 5\% genetic distance criterion proposed by Hellgren et al. (2007), the new haplotype P-BUTBUT02 could be attributed to $P$. circumflexum because of this low genetic distance and tight clustering with known haplotypes. However, to confirm this criterion, it is essential to take morphological measurements and identify sexual development stages with detailed descriptions. In the present study, the prepared films from $B$. buteo and A. nisus blood samples (P-BUTBUT02 haplotypes) were not sufficient for descriptions of parasite morphology due to low intensity infections. Thus, BUTBUT02 could only be identified to the genus level as Plasmodium.

Turkey is located in the Western Palearctic ecozone and has one of the richest bird faunas in this zoogeographic region and is home to two important migration routes that have favourable wetland habitats for migratory birds (Magnin et al. 2000). However, the avian haemosporidian studies are very limited in the vast territories of Turkey. 
Inci et al. (2012) surveyed the lineage diversity of avian haemosporidians in mosquitoes, which were collected from Kayseri Province in the Central Anatolian Region. The authors analysed these mosquitoes using molecular techniques and reported the presence of several avian Plasmodium parasites in potential mosquito vectors in this region.

In the present study, we have described three new avian haplotypes infecting raptor birds, which maybe will be highly useful to future studies of diversity and host specificity on the avian haemosporidian. The injured and/or sick raptor birds examined in this study were also found in Kayseri Province and 32\% of examined birds were infected with haemosporidians. We therefore suggest further investigations on the diversity of avian haemosporidians and on potential host-parasite relationships should be conducted in this region of Turkey.

In conclusion, the findings presented here provide a baseline for a better understanding of the distribution of avian haemosporidian parasites in the poorly studied group of raptor birds in the Central Anatolia Region of Turkey. This study shows that a combination of PCR-based and microscopic methods is an ideal approach for understanding the true diversity and host specificity of these parasites. In addition, our findings open new horizons for the study of avian host interactions among avian haemosporidians. Further studies with a larger sample size in different regions within Turkey may be useful to better understand the epidemiology and ecology of haemosporidian parasites in raptor birds.

Acknowledgements. The authors are grateful staff of the Surgery Department of Veterinary Education Research and Practice Hospital of Erciyes University, Kayseri, Turkey for providing the blood samples used for this study. The authors would also like to thank S. Bensch, Lund University, Department of Biology, Molecular Ecology and Evolution Laboratory, Lund, Sweden for providing positive DNA samples of Leucocytozoon sp. Special thanks are due to anonymous reviewers for their valuable comments that improved this paper. Finally, the authors appreciate the laboratory assistance from G. Yetismis, Z. Kocer and G. Sahingoz Demirpolat.

\section{REFERENCES}

Beadell J.S., Gering E., Austin J., Dumbacher J.P., Peirce M.A., Pratt T.K., Atkinson C.T., Fleischer R.C. 2004: Prevalence and differential host-specificity of two avian blood parasite genera in the Australo-Papuan region. Mol. Ecol. 13: 3829-3844.

Beadell J.S., Ishtiaq F., Covas R., Melo M., Warren B.H., Atrinson C.T., Bensch S., Graves G.R., Jhala Y.V., Peirce M.A., Rahmani A.R., Fonseca D.M., Fleischer R.C. 2006: Global phylogeographic limits of Hawaii's avian malaria. Proc. R. Soc. B. 273: 2935-2944.

Bensch S., Hellgren O., Križanauskienė A., Palinauskas V., Valkiūnas G., Outlaw D., Ricklefs R.E. 2013: How can we determine the molecular clock of malaria parasites? Trends. Parasitol. 29: 363-369.

Bensch S., Hellgren O., Pérez-Tris J. 2009: MalAvi: a public database of malaria parasites and related haemosporidians in avian hosts based on mitochondrial cytochrome $b$ lineages. Mol. Ecol. Resour. 9: 1353-1358.

Bensch S., Stuernman M., Hasselquist D., Ostman O., Hansson B., Westerdahl H., Pinheiro R.T. 2000: Host specificity in avian blood parasites: a study of Plasmodium and Haemoproteus mitochondrial DNA amplified from birds. Proc. R. Soc. Lond. B. 267: 1583-1589.

Bonneaud C., Sepil I., Milá B., Buermann W., Pollinger J., Sehgal R.N.M., Valkiūnas G., Iezhova T.A., SaAtchi S., Sмitн T.B. 2009: The prevalence of avian Plasmodium is higher in undisturbed tropical forests of Cameroon. J. Trop. Ecol. 25: 439-447.

Chagas C.R., Valkiūnas G., Nery C.V., Henrique P.C., GonZalez I.H., Monteiro E.F., Guimarães Lde O., Romano C.M., Kirchgatter K. 2013: Plasmodium (Novyella) nucleophilum from an Egyptian goose in São Paulo Zoo, Brazil: microscopic confirmation and molecular characterization. Int. J. Parasitol. Parasites Wildl. 3: 286-291.

Clark N.J., Adlard R.D., Clegg S.M. 2015: Molecular and morphological characterization of Haemoproteus (Parahaemoproteus) ptilotis, a parasite infecting Australian honeyeaters (Meliphagidae), with remarks on prevalence and potential cryptic speciation. Parasitol. Res. 114: 1921-1928.

Clark N.J., Clegg S.M., Lima M.R. 2014: A review of global diversity in avian haemosporidians (Plasmodium and Haemo- proteus: Haemosporida): new insights from molecular data. Int. J. Parasitol. 44: 329-338.

Dawson R.D., Bortolotti G.R. 2000: Effects of hematozoan parasites on condition and return rates of American kestrels. Auk 117: 373-380.

Dimitrov D., Zehtindjiev P., Bensch S. 2010: Genetic diversity of avian blood parasites in SE Europe: cytochrome b lineages of the genera Plasmodium and Haemoproteus (Haemosporida) from Bulgaria. Acta Parasitol. 55: 201-209.

Forrester D.J., Greiner E.C. 2008: Leucocytozoonosis. In: C.T. Atkinson, N.J. Thomas and D.B. Hunter (Eds.), Parasitic Diseases of Wild Birds. Blackwell Publishing, Ames, Iowa, pp. 54-107.

Fourcade Y., Keišs O., Richardson D.S., Secondi J. 2014: Continental-scale patterns of pathogen prevalence: a case study on the corncrake. Evol. Appl. 7: 1043-1055.

Hanel J., Doležalová J., Stehlíková Š., Modrý D., ChudoBA J., Synek P., VotÝPKA J. 2016: Blood parasites in northern goshawk (Accipiter gentilis) with an emphasis to Leucocytozoon toddi. Parasitol. Res. 115: 263-270.

Hellgren O., Križanauskiené A., Valkiūnas G., Bensch S. 2007: Diversity and phylogeny of mitochondrial cytochrome B lineages from six morphospecies of avian Haemoproteus (Haemosporida: Haemoproteidae). J. Parasitol. 93: 889-896.

Hellgren O., Pérez-Tris J., Bensch S. 2009: A jack-of-alltrades and still a master of some: prevalence and host range in avian malaria and related blood parasites. Ecology. 90: 28402849.

Hellgren O., Waldenström J., Bensch S. 2004: A new PCR assay for simultaneous studies of Leucocytozoon, Plasmodium, and Haemoproteus from avian blood. J. Parasitol. 90: 797-802.

Huelsenbeck J.P., Ronquist F. 2001: MRBAYES: Bayesian inference of phylogenetic trees. Bioinformatics 17: 754-755.

Inci A., Yazar S., Tuncbilek A.S., Canhilal R., Doganay M., Aydin L., Aktas M., Vatansever Z., Ozdarendeli A., Ozbel Y., Yildirim A., Duzlu O. 2013: Vectors and vector-borne diseases in Turkey. Ankara. Univ. Vet. Fak. Derg. 60: 281-296.

Inci A., Yildirim A., Nuabo K.Y., Duzlu O., Biskin Z., CiloGLU A. 2012: Detection and molecular characterization of avian Plasmodium from mosquitoes in central Turkey. Vet. Parasitol. 188: $179-184$ 
Kearse M., Moir R., Wilson A., Stones-Havas S., Cheung M., Sturrock S., Buxton S., Cooper A., Markowitz S., Duran C., Thierer T., Ashton B., Mentuies P., DrumMOND A. 2012: Geneious Basic: an integrated and extendable desktop software platform for the organization and analysis of sequence data. Bioinformatics. 28: 1647-1649.

KimuRA M. 1980: A simple method for estimating evolutionary rate of base substitutions through comparative studies of nucleotide sequences. J. Mol. Evol. 16: 111-120.

Križanauskiené A., Hellgren O., Kosarev V., Sokolov L., Bensch S., Valkiūnas G. 2006: Variation in host specificity between species of avian hemosporidian parasites: evidence from parasite morphology and cytochrome B gene sequences. J. Parasitol. 92: 1319-1324.

Krone O., Waldenström J., Valkiūnas G., Lessow O., Müller K., Iezhova T.A., Fickel J., Bensch S. 2008: Haemosporidian blood parasites in European birds of prey and owls. J. Parasitol. 94: 709-715.

Loiseau C., Iezhova T.A., Valkiūnas G., Chasar A., Hutchinson A., Buermann W., Smith T.B., Sehgal R.N.M. 2010: Spatial variation of haemosporidian parasite infection in African rainforest bird species. J. Parasitol. 96: 21-29.

Magnin G., Eken G., Yarar M. 2000: Turkey. In: M.F. Heath and M.I. Evans (Eds.), Important Bird Areas in Europe: Priority Sites for Conservation. 2: Southern Europe. BirdLife Conservation Series, No. 8, Cambridge, pp. 651-689.

MaLAvi 2015: http://mbio-serv2.mbioekol.lu.se/Malavi, 10/2015.

Martínez J., Martínez-de la Puente J., Herrero J., del Cerro S., Lobato E., Rivero-de Aguilar J., Vásquez R.A., Merino S. 2009: A restriction site to differentiate Plasmodium and Haemoproteus infections in birds: on the inefficiency of general primers for detection of mixed infections. Parasitology 136: 713-722.

Marzal A., Albayrak T. 2012: Geographical variation of haemosporidian parasites in Turkish populations of Krüper's nuthatch Sitta krueperi. J. Ornithol. 153: 1225-1231.

Merino S., Moreno J., Sanz J.J., Arriero E. 2000: Are avian blood parasites pathogenic in the wild? A medication experiment in blue tits (Parus caeruleus). Proc. R. Soc. Lond. B. 267: $2507-2510$.

Özmen Ö., Haligür M., AdAniR R. 2009: Identification of different protozoa species from a common buzzard (Buteo buteo) Turk. J. Vet. Anim. Sci. 33: 257-260.

Özmen Ö., Haligür M., Yukari B.A. 2005: A study on the presence of leucocytozoonosis in wild bird of Burdur district. Turk. J. Vet. Anim. Sci. 29: 1273-1278.

Palinauskas V., Kosarev V., Shapoval A., Bensch S., VALKIŪNAS G. 2007: Comparison of mitochondrial cytochrome $\mathrm{b}$ lineages and morphospecies of two avian malaria parasites of the subgenera Haemamoeba and Giovannolaia (Haemosporida: Plasmodiidae). Zootaxa 1626: 39-50.

Pérez-Rodríguez A., de la Puente J., Onrubia A., PérezTRIS J. 2013: Molecular characterization of haemosporidian parasites from kites of the genus Milvus (Aves: Accipitridae). Int. J. Parasitol. 43: 381-387.

Posada D. 2008: jModelTest: phylogenetic model averaging. Mol. Biol. Evol. 25: 1253-1256.

Remple J.D. 1981: Avian malaria with comments on other haemosporidia in large falcons. In: J.E. Cooper and A.G. Greenwood (Eds.), Recent Advances in the Study of Raptor Diseases. Chiron Publications, Keighley, West Yorkshire, pp. 107-110.

Remple J.D. 2004: Intracellular Hematozoa of raptors: a review and update. J. Avian. Med. Surg. 18: 75-88.
Ricklefs R.E., Fallon S.M. 2002: Diversification and host switching in avian malaria parasites. Proc. R. Soc. Lond. B 269: $885-892$.

Ricklefs R.E., Fallon S.M., Bermingham E. 2004: Evolutionary relationships, cospeciation and host switching in avian malaria parasites. Syst. Biol. 53: 111-119.

Sehgal R.N.M., Hull A.C., Anderson N.L., Valkiūnas G., Markovets M.J., Kawamura S., Tell L.A. 2006: Evidence for cryptic speciation of Leucocytozoon spp. (Haemosporida, Leucocytozoidae) in diurnal raptors. J. Parasitol. 92: 375-379.

Tamura K., Stecher G., Peterson D., Filipski A., Kumar S. 2013: MEGA6: Molecular Evolutionary Genetics Analysis version 6.0. Mol. Biol. Evol. 30: 2725-2729.

VAlKIŪNAs G. 2005: Avian Malaria Parasites and Other Haemosporida. CRC Press, Boca Raton, Florida, 932 pp.

Valkiūnas G., AtKinson C.T., Bensch S., Sehgal R.N.M., Ricklefs R.E. 2008b: Parasite misidentifications in GenBank: how to minimize their number? Trends. Parasitol. 24: 247-248.

Valkiūnas G., Bensch S., Iezhova T.A., Križanauskienė A., Hellgren O., Bolshakov C.V. 2006: Nested cytochrome b polymerase chain reaction diagnostics underestimate mixed infections of avian blood haemosporidian parasites: microscopy is still essential. J. Parasitol. 92: 418-422.

Valkiūnas G., Iezhova T.A., Križanauskiené A., Palinauskas V., Sehgal R.N.M., Bensch S. 2008a: A comparative analysis of microscopy and PCR-based detection methods for blood parasites. J. Parasitol. 94: 1395-1401.

Valkiūnas G., Iezhova T.A., Loiseau C., Sehgal R.N.M. 2009: Nested cytochrome b polymerase chain reaction diagnostics detect sporozoites of haemosporidian parasites in peripheral blood of naturally infected birds. J. Parasitol. 95: 1512-1515.

Valkiūnas G., Palinauskas V., Ilgūnas M., Bukauskaitė D., Dimitrov D., Bernotiené R., Zehtindjiev P., Ilieva M., IEzhova T.A. 2014: Molecular characterization of five widespread avian haemosporidian parasites (Haemosporida), with perspectives on the PCR-based detection of haemosporidians in wildlife. Parasitol. Res. 113: 2251-2263.

Ventim R., Morais J., Pardal S., Mendes L., Ramos J.A., Perez-Tris J. 2012: Host-parasite associations and host-specificity in haemoparasites of reed bed passerines. Parasitology 139: $310-316$.

Waldenström J., Bensch S., Hasselquist D., Ostman O. 2004: A new nested polymerase chain reaction method very efficient in detecting Plasmodium and Haemoproteus infections from avian blood. J. Parasitol. 90: 191-194.

Waldenström J., Bensch S., Kiboi S., Hasselquist D., OtTOSSON U. 2002: Cross-species infection of blood parasites between resident and migratory songbirds in Africa. Mol. Ecol. 11: $1545-1554$.

Yildirim A., Aysul N., Bayramli G., Inci A., Eren H., Aypak S., Duzlu O., Ciloglu A., Onder Z. 2013: Detection and molecular characterization of a Haemoproteus lineage in a tawny owl (Strix aluco) in Turkey. Ankara. Univ. Vet. Fak. Derg. 60: 179-183.

Yigit N., Saygili F., Colak E., Sozen M., Karatas A. 2008: [Ornithology "Bird Science" Lecture Notes.] PRD, Ankara, 371 pp. (In Turkish.)

Zehtindjiev P., Ivanova K., Mariaux J., Georgiev B.B. 2013: First data on the genetic diversity of avian haemosporidians in China: cytochrome b lineages of the genera Plasmodium and Haemoproteus (Haemosporida) from Gansu Province. Parasitol. Res. 112: 3509-3515. 